\title{
Fallout aus chinesischen Kernwaffenversuchen
}

\author{
J. Fahland
}

Institut für Anorganische Chemie und Kernchemie der Universität Mainz

(Z. Naturforsch. 24 a, 653-659 [1969] ; eingegangen am 31. Januar 1969)

\begin{abstract}
Short-lived radionuclides have been measured in fallout of Chinese nuclear tests. The measured yield ratios of the nuclides ${ }^{95} \mathrm{Zr},{ }^{111} \mathrm{Ag},{ }^{115} \mathrm{Cd},{ }^{156} \mathrm{Eu},{ }^{237} \mathrm{U}$ and ${ }^{239} \mathrm{~Np}$ have been corrected for fractionation effects and compared to calculated ratios. Free parameters of the calculation such as most frequent neutron energy, time integrated neutron flux, and fission material were adjusted by means of a least square criterion. For the tests $2,3,5$ and 7 the most frequent neutron energy was found to be $5 \mathrm{keV}$. The corresponding Maxwellian neutron fluxes were within $10^{21}$ and $10^{24} \mathrm{n} / \mathrm{cm}^{2}$. In addition, 14-MeV neutron fluxes within $10^{19}$ and $10^{22} \mathrm{n} / \mathrm{cm}^{2}$ had to be assumed. The analysis yields as average of the converted fission material a mixture of ${ }^{235} \mathrm{U}$ and ${ }^{238} \mathrm{U}$ with $7-99 \%$ of ${ }^{235} \mathrm{U}$. - The fractionation of ${ }^{140} \mathrm{Ba}$ in surface bursts corresponded to that in air bursts.
\end{abstract}

Die Kernwaffenversuche führten nicht nur zu umfangreichen Messungen über die Verbreitung strahlenbiologisch toxischer Radionuklide ${ }^{1,2}$, sondern auch zu neuen Erkenntnissen über Kondensationsvorgänge bei hohen Temperaturen ${ }^{3-6}$, Luftströmungen in der Tropo- und Stratosphäre ${ }^{7-12}$, die Elementsynthese ${ }^{13}$ und Kernreaktionen bei extrem hohen Neutronenflüssen ${ }^{14-17}$.

Für einige chinesische Kernwaffenversuche wurde aus der Falloutzusammensetzung auf das Spalt. material geschlossen ${ }^{18-21}$. In der vorliegenden Arbeit soll gezeigt werden, daß solche Schlüsse eine detaillierte Analyse voraussetzen, in der nach einer Korrektur der Fraktionierungseffekte die Parameter ,wahrscheinliche Neutronenenergie, zeitintegrierter Neutronenfluß und Spaltmaterial" solange variiert werden, bis eine optimale Übereinstimmung zwischen gemessenen und berechneten Werten vorliegt. Folgendes Modell der Kernwaffenexplosion wird zugrunde gelegt: Die überkritische

1 U. S. Atomic Energy Comm., New York, Health and Safety Lab., HASL circular.

2 J. L. Kulp u. A. R. Schulert, Strontium-90 in Man and His Environment, Geochemical Lab., Lamont Geological Observatory, Columbia University, Palisades, New York 1962.

3 E. C. Freiling, Science 133, 1991 [1961].

4 E. C. Freiling u. M. A. Kay, Nature 209, 236 [1966].

5 R. G. BEDFord u. D. D. JACKson, University of California, UCRL-12314 [1965].

6 C. F. Miller, U. S. Naval Radiological Defense Lab., San Francisco, Calif., USNRDL-TR-425 [1960].

7 P. W. Krey u. W. C. Rosa, U.S. Atomic Energy Comm., New York, Health and Safety Lab., HASL-161, 288 [1965].

8 W. W. Cooper u. P. K. Kuroda, J. Geophys. Res. 71, $5471[1966]$.

9 J. O. Baugh, K. Yoshikawa u. P. K. Kuroda, Science 155, 1405 [1967].

10 M. N. Rao u. K. K. Menon, Nature 213, 26 [1967].
Anordnung des Spaltmaterials löst neutroneninduzierte Kernspaltung aus, deren Energie eine genügend hohe Temperatur erzeugt, um Kernverschmelzung durch (D,T)-Reaktion einzuleiten. Die entstehenden $14 \mathrm{MeV}$-Neutronen bewirken weitere Kernspaltung. Spaltprodukte und induzierte Radionuklide werden zeitlich nacheinander in zwei Stufen gebildet, zuerst mit Spaltneutronen und anschließend mit Fusionsneutronen. Für die Bildungsgesetze der Radionuklide werden drei Annahmen gemacht:

1. Die Fraktionierungseffekte werden durch Korrektur eliminiert.

2. Die Neutroneneinfangreaktion der Spaltprodukte ist vernachlässigbar, so daß durch diesen Sekundärprozeß Spaltprodukte weder verbraucht noch gebildet werden.

3. Das Neutronenspektrum wird durch ein Maxwell-Spektrum plus einem Anteil $14 \mathrm{MeV}$-Neutronen approximiert. Das Maxwell-Spektrum enthält

11 D. H. Peirson u. R. S. Cambray, Nature 216, 755 [1967].

12 P. Kruger u. A. Miller, J. Geophys. Res. 69, 1469 [1964].

13 G. I. Bell, Rev. Mod. Phys. 39, 59 [1967].

14 A. G. W. Cameron, Canad. J. Phys. 37, 322 [1959].

15 G. I. Bell, Phys. Rev. 139 A, 1207 [1965].

16 Los Alamos Radiochemistry Group, Phys. Rev. Letters 14, 962 [1965].

17 D. W. Dorn u. R. W. Hoff, Phys. Rev. Letters 14, 440 [1965].

18 K. G. Vohra, Government of India, Atomic Energy Comm., Atomic Energy Establishment Trombay, Bombay, AEET-240 [1965].

19 P. K. Kuroda, B. D. Palmer, M. Attrep, J. N. Beck, R. Ganapathy, D. D. Sabu u. M. N. RaO, Science 147, 1284 [1965].

20 M. N. RaO, K. Yoshikawa, D. D. Sabu, R. Clarke u. P. K. Kuroda, Science 153, 633 [1966].

21 O. C. Allkofer, H. Hauser, H. Meyerbröker, M. Simon u. C. Wöhren, Z. Naturforsch. 23 a, 1272 [1968]. 
die moderierten Spalt- und Fusionsneutronen, während der $14 \mathrm{MeV}$-Anteil ungebremste Neutronen der $(\mathrm{D}, \mathrm{T})$-Reaktion berücksichtigt.

Für die vergleichende Analyse eignen sich nicht alle Radionuklide. Erstens muß die Halbwertszeit mindestens einige Tage betragen, damit das Nuklid überhaupt erfaßt werden kann; sie darf aber einige Wochen nicht übersteigen, damit aus älteren Explosionen stammender Fallout die Ergebnisse nicht verfälscht. Zweitens muß die Fraktionierung, d.h. die von der Umgebung der Explosion abhängige Verschiebung der ursprünglichen Falloutzusammensetzung, berücksichtigt werden können, wozu die betreffende Fraktionierungskorrelation benötigt wird; diese Beziehungen gibt FreILING 3,4 an. Günstig für eine solche Analyse sind die Radioisotope ${ }^{111} \mathrm{Ag},{ }^{115} \mathrm{Cd},{ }^{156} \mathrm{Eu},{ }^{237} \mathrm{U}$ und ${ }^{239} \mathrm{~Np}$, weil deren Ausbeuten stark von der Neutronenenergie und vom Spaltmaterial abhängen. Zusätzlich braucht man für die Fraktionierungskorrektur ${ }^{89} \mathrm{Sr}$ (oder ${ }^{140} \mathrm{Ba}$ ) sowie ${ }^{95} \mathrm{Zr}$ (oder ${ }^{147} \mathrm{Nd},{ }^{153} \mathrm{Sm}$ ).

Der Vergleich gemessener und berechneter Ausbeutequotienten muß dann zeigen, ob die resultierenden Parameter der Falloutzusammensetzung - Neutronenspektrum, Neutronenfluß, Spaltmaterial - mit den bisher bekannten Ergebnissen in Einklang stehen.

\section{Experimentelles}

Flugzeuge adsorbieren den Fallout an der Oberfläche ${ }^{22}$. Das radioaktive Material wird durch Abwischen mit Filtrierpapier von den Flugzeugen entnommen, bei $500^{\circ} \mathrm{C}$ verascht, durch eine Soda-Borax-Schmelze aufgeschlossen und in 6 - $\mathrm{m} . \mathrm{HCl}$ gelöst.

Die radiochemischen Verfahren für die Isolierung von $\mathrm{Ba}, \mathrm{Sr}, \mathrm{Zr}, \mathrm{Ag}$ und $\mathrm{Cd}$ sind Standardverfahren ${ }^{23}$. Für die Trennung von U, Np, Sm und Eu wird die Säulenextraktionschromatographie ${ }^{24-26}$ benutzt unter Verwendung von Tri-n-butylphosphat (TBP), Tri-n-octylamin (TOA) und Di-(2-äthylhexyl)-phosphorsäure (HDEHP) als Extraktionsmittel, welches auf Polytetrafluoräthylen-Pulver aufgezogen ist. Die Aufschlußlösung wird über eine TOA-Säule gegeben. Dabei werden $\mathrm{U}, \mathrm{Np}, \mathrm{Cd}, \mathrm{Zr}$ und Fe extrahiert,

22 O. C. Allkofer, E. Bagge u. J. Trümper, Atomkernenergie 7, 173, 204, 249 [1962].

23 C. D. Coryell u. N. Sugarman, Radiochemical Studies - The Fission Products, McGraw Hill, New York 1961.

24 H. Eschrich, Kjeller Research Establishment, Norway, KR-11 [1961].

25 R. Denig, N. Trautmann u. G. Herryanx, Symposium ..Reaktorchemie" der Fachgruppe Kern-, Radio- u. Strahlenchemie in der Gesellschaft Deutscher Chemiker, Jülich 3. -4. 10. 1966.

26 E. Cerrai, C. Testa u. C. Triulzi, Energia Nucleare 9, 193, 377 [1962]. während $\mathrm{Ba}, \mathrm{Sr}, \mathrm{Ag}$ und $\mathrm{SE}$ (Seltene Erden) nebst komplex gelöster $\mathrm{SiO}_{2}$ durchlaufen. $\mathrm{U}, \mathrm{Np}, \mathrm{Zr}$ und $\mathrm{Fe}$ werden mit 0,1-m. $\mathrm{HCl}$ eluiert. Anschließend werden $\mathrm{U}, \mathrm{Np}$ und $\mathrm{Zr}$ aus 8-m. $\mathrm{HNO}_{3}$ in einer TBP-Säule extrahiert; Fe läuft durch. Nach Elution des $\mathrm{U}, \mathrm{Np}$ und $\mathrm{Zr}$ mit $0,1-\mathrm{m} \cdot \mathrm{HNO}_{3}$ werden $\mathrm{Np}$ und $\mathrm{Zr}$ aus $6-\mathrm{m} . \mathrm{HCl} / \mathrm{SO}_{2}$ in einer HDEHP-Säule extrahiert; das U läuft durch. Schließlich werden $\mathrm{Np}$ und $\mathrm{Zr}$ getrennt ${ }^{23}$. - Aus dem Gemisch Ba, Sr, Ag, SE wird durch Verdünnen $\mathrm{AgCl}$ gefällt. Im Filtrat wird die Kieselsäure abgeschieden; anschließend trennt man die SE an einer weiteren HDEHP-Säule ${ }^{26}$. Für die Bestimmung von Ba, $\mathrm{Sr}$ und $\mathrm{Cd}$ wurden gesonderte Einwaagen gemacht.

Die chemische Ausbeute wird durch zugesetzte Trägerelemente kontrolliert; für Uran dient ${ }^{235} \mathrm{U}$, für Neptunium ${ }^{237} \mathrm{~Np}$ als Indikator, der aktivierungsanalytisch bestimmt wird.

Die Messung der Radioaktivität folgt mit einem geeichten $\mathrm{NaJ}(\mathrm{Tl})-$ Vielkanalimpulshöhenanalysator ${ }^{27}$ und mit geeichten Antikoinzidenzzählern 28. Zur Reinheitskontrolle werden Zerfallskurven oder Gammaspektren gemessen.

\section{Meßergebnisse}

Die Anfangsaktivitäten der untersuchten Radionuklide gibt Tab. 1. Diese Tabelle zeigt, daß die spezifische Aktivität an kurzlebigen Spaltprodukten, wie ${ }^{140} \mathrm{Ba}$, im Fallout der Explosion vom 17. 6. 1967 etwa 100mal kleiner war als in den anderen Proben, vermutlich weil der Feuerball mit dem Fallout durch den hohen Explosionswert der 3 Megatonnen-Wasserstoffbombe etwa $30 \mathrm{~km}$ in die Stratosphäre aufstieg ${ }^{29}$ und die Explosion obendrein in großer Höhe stattfand 30,31 , so daß das Absinken der Falloutpartikel bis auf die Höhe der Flugzeugrouten $(9-13 \mathrm{~km})$ sehr stark verzögert wird. Bei schwächeren Explosionen steigt der Feuerball nicht so hoch. Entsprechend werden teilweise sehr hohe spezifische Aktivitäten an frischem Fallout gemessen.

\section{Fraktionierung des Fallout}

Etwa 30-40 sec nach einer Kernwaffenexplosion $^{3,32,33}$ kondensieren aus der verdampften

27 H. Mundschenk, Nucl. Instr. Methods 44, 325; 45, 77; 45, 261 [1966]; Intern. J. Appl. Radiation Isotopes 18, 365 [1967].

28 B. P. Bayhurst u. R. J. Prestwood, Nucleonics 17, No. 3,82 [1959].

29 S. GLasstone, Die Wirkungen der Kernwaffen, C. Heymanns Verlag KG, Köln, 1964, S. 34, Bild 2.16; S. 372.

30 A. Bärwolf, Die Welt, 7. Aug. 1967.

31 Kerntechnik 9, 330 [1967].

32 Arkansas University, Fayetteville, Department of Chemistry, ORO-2529-6 [1966].

33 T. Mamuro, T. Matsunami u. A. Fujita, Health Phys. 14, 223 [1968]. 


\begin{tabular}{|c|c|c|c|c|c|c|c|}
\hline \multicolumn{3}{|c|}{$\begin{array}{c}\text { Explosion Nr. } \\
\text { Datum } \\
\text { g Asche } \\
\text { Nuklid }\end{array}$} & \multirow{2}{*}{$\begin{array}{c}2 \\
\begin{array}{c}14.5 .65 \\
0,5 \\
\operatorname{Imp} / \min \end{array} \\
3,75 \cdot 10^{4}\end{array}$} & \multirow{2}{*}{$\begin{array}{c}3 \\
9.5 .66 \\
1,8 \\
\operatorname{Imp} / \min \\
8,29 \cdot 10^{4}\end{array}$} & \multirow{2}{*}{$\begin{array}{c}5 \\
28.12 .66 \\
0,5 \\
\operatorname{Imp} / \min \\
-\end{array}$} & \multirow{2}{*}{$\begin{array}{c}6 \\
17.6 .67 \\
22,0 \\
\operatorname{Imp} / \mathrm{min} \\
1,00 \cdot 10^{6 *}\end{array}$} & \multirow{2}{*}{$\begin{array}{c}7 \\
24.12 .67 \\
3,0 \\
\text { Imp/min } \\
-\end{array}$} \\
\hline $\mathrm{Sr}$ & 89 & $50,5 \mathrm{~d}$ & & & & & \\
\hline $\mathrm{Sr}$ & 90 & 27,7 a & $1,06 \cdot 10^{5 *}$ & $1,40 \cdot 10^{5 *}$ & - & $7,50 \cdot 10^{5 *}$ & - \\
\hline $\mathrm{Zr}$ & 95 & $65,0 \mathrm{~d}$ & $5,00 \cdot 10^{4}$ & $3,27 \cdot 10^{5}$ & $5,58 \cdot 10^{4}$ & $2,22 \cdot 10^{5 *}$ & $4,48 \cdot 10^{5 *}$ \\
\hline $\mathrm{Ag}$ & 111 & $7,5 \mathrm{~d}$ & - & $1,54 \cdot 10^{4}$ & $1,58 \cdot 10^{4}$ & $4,75 \cdot 10^{3}$ & $8,50 \cdot 10^{3}$ \\
\hline $\mathrm{Cd}$ & 115 & $2,2 \mathrm{~d}$ & - & - & - & - & $2,52 \cdot 10^{4}$ \\
\hline $\mathrm{Ba}$ & 140 & $12,8 \mathrm{~d}$ & $2,42 \cdot 10^{5}$ & $6,20 \cdot 10^{5}$ & $6,49 \cdot 10^{5}$ & $6,01 \cdot 10^{4}$ & $2,64 \cdot 10^{5}$ \\
\hline $\mathrm{Nd}$ & 147 & $11,1 \mathrm{~d}$ & $2,96 \cdot 10^{5}$ & - & - & $2,44 \cdot 10^{4}$ & $8,20 \cdot 10^{4}$ \\
\hline $\mathrm{Sm}$ & 153 & $46,7 \mathrm{~h}$ & - & - & - & - & $2,60 \cdot 10^{4}$ \\
\hline $\mathrm{Eu}$ & 156 & $15,2 \mathrm{~d}$ & - & - & - & - & $4,06 \cdot 10^{2}$ \\
\hline $\mathrm{U}$ & 237 & $6,75 \mathrm{~d}$ & - & $2,80 \cdot 10^{6}$ & $9,40 \cdot 10^{4}$ & - & $3,33 \cdot 10^{5}$ \\
\hline $\mathrm{Np}$ & 239 & $2,35 \mathrm{~d}$ & - & - & $6,39 \cdot 10^{6}$ & - & $3,19 \cdot 10^{7}$ \\
\hline
\end{tabular}

Tab. 1. Anfangsaktivitäten von Fallout.

* Die indizierten Radionuklide stammen hauptsächlich aus altem Fallout. Aus Sm-153 und/oder Nd-147 wurden für Test 6 und 7 die äquivalenten Aktivitäten Zr-95 berechnet: 1,22 $10^{4}$, bzw. 3,21 $10^{4} \mathrm{Imp} / \mathrm{min}$. Die Umrechnung erfolgte mit Gl. (1).

Masse Partikel, an denen sich die Spaltprodukte, ihrem Dampfdruck entsprechend 5,3,4 anlagern. Die Folge davon ist eine frühzeitige Adsorption schwerflüchtiger Spaltprodukte an größeren Partikeln bei hoher Temperatur, während die verbleibenden flüchtigen Spaltprodukte später bei niedriger Temperatur an kleineren Partikeln adsorbiert werden. Dieser Fraktionierungsprozeß ist bei Explosionen an der Erdoberfläche stärker ausgeprägt als bei Explosionen in der Luft, weil die beteiligten inaktiven Massen stark differieren ${ }^{35,36}$. Gut untersucht ist der Fraktionierungsprozeß für Explosionen in der Luft, während für Explosionen über Silikatböden nur wenig Daten existieren ${ }^{37}$. Die starke Fraktionierung des Fallouts von chinesischen Kernwaffenversuchen wird vielfach berichtet ${ }^{38-42}$.

Um gemessene Ausbeutequotienten hinsichtlich der Fraktionierungseffekte zu korrigieren, müssen hier wegen des fehlenden Zahlenmaterials für Bodenoberflächenexplosionen die Fraktionierungsdaten für Explosionen in der $\mathrm{Luft}^{3-5}$ benutzt werden. Nach Freiling ${ }^{3,4}$ gilt die Beziehung:

$$
\frac{N_{\mathrm{i}}}{N_{\mathrm{Zr}-95}} \cdot \frac{Y_{\mathrm{Zr}-95}}{Y_{\mathrm{i}}}=\left(\frac{N_{\mathrm{Sr}-89}}{N_{\mathrm{Zr}-95}} \cdot \frac{Y_{\mathrm{Zr}-95}}{Y_{\mathrm{Sr}-89}}\right)^{m_{\mathrm{i}}}
$$

34 J. H. Norman, General Dynamics Corp. San Diego, Calif., General Atomics Div., GA-7058 [1966].

35 J. SisEFski, Nature 210, 1143 [1966].

36 G. Persson, Nature 209, 1193 [1966].

37 G. R. Crocker u. E. C. Freiling, Vortrag 80, 115 th National Meeting American Chemical Soc., San Francisco, April 1968.

38 F. G. Gioncelli u. A. A. Cigna, J. Fis. Prot. Radiaz. 10, 192 [1966]. oder abgekürzt

$$
r_{\mathrm{i}, 95}=\left(r_{89,95}\right)^{m_{1}} .
$$

Es bedeuten: $N_{\mathrm{i}}=$ gemessene Zahl der Atome des Nuklides i; $Y_{\mathrm{i}}=$ kumulierte Spaltausbeute des Nuklids i; $m_{\mathrm{i}}=$ Steigung der log-log-Fraktionierungsgeraden für das Nuklid i. Freiling 3,4 benutzte die Spaltausbeuten für ${ }^{235} \mathrm{U}$-Spaltung mit thermischen Neutronen nach $\mathrm{K}_{\text {ATCOFF }}{ }^{43}$, während in dieser Arbeit neuere Zahlenwerte ${ }^{44}$ eingesetzt werden: $Y_{\mathrm{Zr}-95} / Y_{\mathrm{Sr}-89}=1,21$ und $Y_{\mathrm{Zr}-95} / Y_{\mathrm{Ba}-140}$ $=1,074$. Das Verhältnis $Y_{\mathrm{Zr}-95} / Y_{\mathrm{Ba}-140}$ ist für verschiedene Neutronenenergien und Spaltmaterialien auf etwa $10 \%$ konstant $^{3}$ und beeinflußt die weitere Auswertung hinsichtlich des Spaltmaterials und der Neutronenenergie nicht. Der Arbeit von FreILING 3,4 wurde entnommen: $m_{\mathrm{Ag}-111}, m_{\mathrm{Cd}-115}$, $m_{\mathrm{U}-237}, m_{\mathrm{Np}-239}=0,80$ und $m_{\mathrm{Ba}-140}=0,62$.

Zur Prüfung, ob die Fraktionierungskorrelation Gl. (1) auch für Explosionen über Silikatböden gilt, wurden in Abb. 1 die wenigen verfügbaren Daten über die Fraktionierung des ${ }^{140} \mathrm{Ba}$, bezogen auf ${ }^{95} \mathrm{Zr}$ und ${ }^{89} \mathrm{Sr}$, mit der von Freiling ${ }^{3}, 4$ gemessenen Fraktionierungsgeraden verglichen, welche für Explosionen in der Luft gilt. Wie die Übereinstimmung

39 T. Sotobayashi, T. Suzuki u. S. Koyama, Bull. Chem. Soc. Japan 40, 1555 [1967].

40 A. Aarkrog u. J. Lippert, Nature 213, 1001 [1967].

41 G. Persson, Nature 209, 1228 [1966].

42 T. Mamuro u. A. Fujita, Health Phys. 13, 197 [1967].

43 S. Katcoff, Nucleonics 16, No. 4, 78 [1958].

44 G. Herrmans, Habilitationsschrift, Mainz 1961. 
der Meßwerte mit der Geraden in Abb. 1 zeigt, fraktioniert ${ }^{140} \mathrm{Ba}$ bei Explosionen über Silikatböden mit dem für Luft gültigen Fraktionierungs-

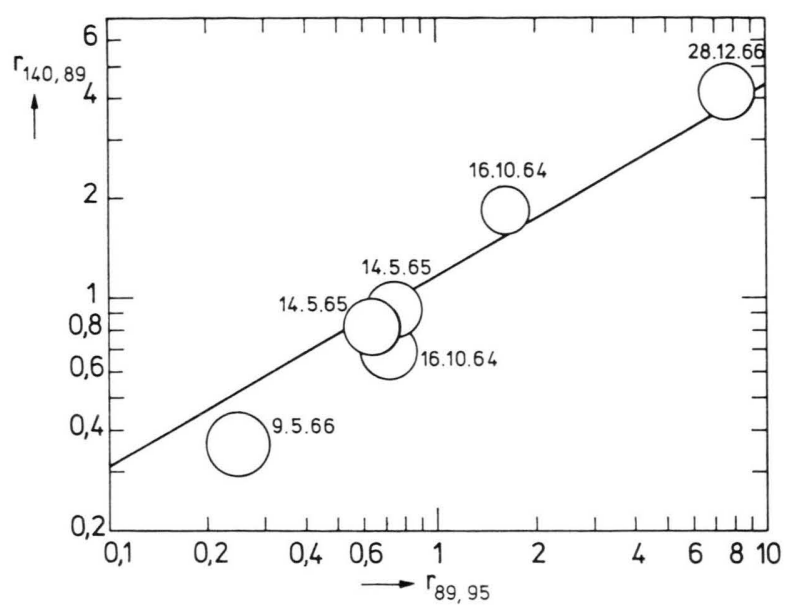

Abb. 1. Die Fraktionierung des ${ }^{140} \mathrm{Ba}$ in Explosionen an der Erdoberfläche wird mit der von FreILING ${ }^{3}, 4$ gemessenen Fraktionierung bei Explosionen in der Luft (Gerade) verglichen. Die Daten vom 16.10.64 und 14.5.65 stammen teilweise von VoHRA ${ }^{18}$ und Kuroda ${ }^{19}$. Statt $r{ }_{140,89}$ lies $r_{140,95}$.

parameter $m_{\mathrm{Ba}-140}$. Dasselbe für ${ }^{111} \mathrm{Ag},{ }^{115} \mathrm{Cd}$, ${ }^{237} \mathrm{U}$ und ${ }^{239} \mathrm{~Np}$ vorausgesetzt, erhält man für die Korrektur dieser Meßdaten aus Gl. (1):

$$
\begin{aligned}
\frac{Y_{\mathrm{Ag}-111}}{Y_{\mathrm{Zr}-95}}=\frac{N_{\mathrm{Ag}-111}}{N_{\mathrm{Zr}-95}} \cdot\left(\frac{N_{\mathrm{Sr}-89}}{N_{\mathrm{Zr}-95}} \cdot \frac{Y_{\mathrm{Zr}-95}}{Y_{\mathrm{Sr}-89}}\right)^{-m_{\mathrm{Ag}-111}} \\
Y_{\mathrm{Ag}-111}=\frac{N_{\mathrm{Ag}-111}}{N_{\mathrm{Zr}-95}} \cdot\left(\frac{N_{\mathrm{Ba}-140}}{N_{\mathrm{Zr}-95}} \cdot \frac{Y_{\mathrm{Zr}-95}}{Y_{\mathrm{Ba}-140}}\right)^{-m_{\mathrm{Ag}-111} / m_{\mathrm{Ba}-140}}
\end{aligned}
$$

Der potenzierte Faktor in den Gln. (2)-(3) ist die Korrektur $f_{i}$ für die gemessenen Ausbeutequotienten des ${ }^{111} \mathrm{Ag},{ }^{115} \mathrm{Cd},{ }^{237} \mathrm{U}$ und ${ }^{239} \mathrm{~Np}$ gegenüber ${ }^{95} \mathrm{Zr}$. Die Korrektur kann wahlweise über ${ }^{89} \mathrm{Sr}$ oder ${ }^{140} \mathrm{Ba}$ sowie ${ }^{95 \mathrm{Zr}}$ vorgenommen werden. Innerhalb einer Probe sollten die Korrekturfaktoren $f_{89}$ und $f_{140}$ gleich groß sein. Wieweit das stimmt, zeigt
Tab. 2. Danach ist die mittlere Korrektur für Fallout der Wasserstoffbombenexplosion vom 17.6. 1967 gleich eins, d.h. diese Probe war nicht fraktioniert, sicherlich weil die Explosion in großer Höhe erfolgte. In den übrigen Falloutproben tritt sowohl Anreicherung $f_{\mathrm{i}}<1$ als auch Abreicherung $f_{\mathrm{i}}>1$ auf.

\section{Auswertung, Ergebnisse und Diskussion}

Die gemessenen und die für Fraktionierung korrigierten Ausbeutequotienten sind in Tab. 3 zusammengestellt. Für ${ }^{111} \mathrm{Ag} /{ }^{95} \mathrm{Zr}$ resultiert im Mittel ein korrigiertes Ausbeuteverhältnis von 0,012, das ${ }^{239} \mathrm{Pu}$ als Spaltmaterial ausschließt; denn mit ${ }^{239} \mathrm{Pu}$ als Spaltmaterial müßte dieser Quotient mindestens 0,049 betragen. Somit kann sich die weitere Analyse auf $235 \mathrm{U}$ und ${ }^{238} \mathrm{U}$ als Spaltmaterial beschränken.

Ebenfalls in Tab. 3 aufgeführt sind nach Gl. (7) berechnete Ausbeutequotienten. Ausgewählt ist diejenige Kombination der Variablen, welche ein Fehlerminimum nach Gl. (8) ergibt. Variiert wurden: Die häufigste Neutronenenergie $E_{\mathrm{p}}$ eines Maxwell-Neutronenspektrums zwischen 0,5 und $667 \mathrm{keV}$, wobei $667 \mathrm{keV}$ dem unmoderierten Spaltneutronenspektrum entspricht; der zeitintegrierte Fluß $\Phi t$ dieses Spektrums zwischen $10^{20}$ und $10^{25} \mathrm{n} / \mathrm{cm}^{2}$; der zeitintegrierte Fluß $\varphi \tau$ an $14 \mathrm{MeV}$ Neutronen zwischen Null und $10^{25} \mathrm{n} / \mathrm{cm}^{2}$; und der Beitrag $Q$ des ${ }^{235} \mathrm{U}$ zum Reaktionsablauf zwischen 0,72 und $99,9 \%$, d.h. zwischen dem Anteil des ${ }^{235} \mathrm{U}$ in Natururan und reinem ${ }^{235} \mathrm{U}$. Dieser Beitrag $Q$ ist über den umgesetzten Teil der Waffe gemittelt. Bestand diese z.B. aus 90-proz. ${ }^{235} \mathrm{U}$ mit einem Natururan-Reflektor, so resultiert $Q=90 \%$, wenn der Reflektor nicht merklich umgesetzt wurde.

Korrigierte und berechnete Ausbeutequotienten stimmen innerhalb der zu erwartenden Grenzen Meßfehler $20 \%$, Fraktionierungskorrektur Fehler-

\begin{tabular}{|c|c|c|c|c|c|c|c|c|}
\hline Nr. & $\begin{array}{c}\text { Stärke } \\
\text { kt }\end{array}$ & Typ & Referenz & $N_{89} / N_{95}$ & $N_{140} / N_{95}$ & $f_{89}$ & $f_{140}$ & Mittel \\
\hline 1 & 30 & Boden & 19 & $1,26 \pm 0,26$ & $1,89 \pm 0,40$ & 0,7 & 0,4 & 0,6 \\
\hline 1 & 30 & Boden & 18 & $0,55 \pm 0,11$ & $0,70 \pm 0,14$ & 1,4 & 1,5 & 1,5 \\
\hline 2 & 30 & Boden & 18 & $0,49 \pm 0,10$ & $0,83 \pm 0,17$ & 1,5 & 1,2 & 1,4 \\
\hline 3 & 300 & Boden & Diese Arbeit & $0,19 \pm 0,04$ & $0,37 \pm 0,07$ & 3,2 & 3.3 & 3,3 \\
\hline 5 & 300 & Boden & Diese Arbeit & 二 & $2.30 \neq 0,46$ & - & 0.3 & 0,3 \\
\hline 6 & 3000 & Luft & Diese Arbeit & - & $0,97 \pm 0,19$ & - & 1,0 & 1,0 \\
\hline 7 & 30 & Boden & Diese Arbeit & - & $1,62 \pm 0,32$ & - & 0,5 & 0,5 \\
\hline
\end{tabular}
faktor 2 - überein.

Tab. 2. Fraktionierungskorrekturfaktoren nach Gln. (2)-(3) für die relativen Ausbeuten von Ag-111, Cd-115, U-237 und $\mathrm{Np}-239$. 


\begin{tabular}{|c|c|c|c|c|c|}
\hline Test-Nr. & & 2 & 3 & 5 & $7 * * *$ \\
\hline$Y_{\mathrm{Ag}-111} / Y_{\mathrm{Zr}-95}$ & $\begin{array}{l}\text { gem. } \\
\text { korr. } \\
\text { ber. }\end{array}$ & $\begin{array}{l}0,005 * \\
0,007 \\
0,007\end{array}$ & $\begin{array}{l}0,005 \\
0,017 \\
0,018\end{array}$ & $\begin{array}{l}0,033 \\
0,010 \\
0,007\end{array}$ & $\begin{array}{l}0,031 \\
0,016 \\
0,015\end{array}$ \\
\hline $\mathrm{Y}_{\mathrm{U}-237} / \mathrm{Y}_{\mathrm{Zr}-95}$ & $\begin{array}{l}\text { gem. } \\
\text { korr. } \\
\text { ber. }\end{array}$ & $\begin{array}{l}0,22 * \\
0,31 \\
0,29\end{array}$ & $\begin{array}{l}0,83 \\
2,7 \\
2,4\end{array}$ & $\begin{array}{l}0,16 \\
0,05 \\
0,05\end{array}$ & $\begin{array}{l}1,1 \\
0,5 \\
0,6\end{array}$ \\
\hline $\mathrm{Y}_{\mathrm{Np}-239} / \mathrm{Y}_{\mathrm{Zr}-95}$ & $\begin{array}{l}\text { gem. } \\
\text { korr. } \\
\text { ber. }\end{array}$ & $\begin{array}{l}0,04 * \\
0,06 \\
0,07\end{array}$ & $\begin{array}{l}12 * * \\
40 \\
45\end{array}$ & $\begin{array}{l}7,2 \\
2,2 \\
1,3\end{array}$ & $\begin{array}{l}36 \\
18 \\
16\end{array}$ \\
\hline $\begin{array}{l}\text { Neutronenenergie } E_{\mathrm{p}} \\
\text { Maxwell-Neutronenfluß } \\
14 \mathrm{MeV} \text {-Neutronenfluß } \\
N_{\mathbf{z 3 5}}^{0} /\left(N_{235}^{0}+N_{238}^{0}\right)\end{array}$ & $\begin{array}{l}\mathrm{keV} \\
\mathrm{n} / \mathrm{cm}^{2} \\
\mathrm{n} / \mathrm{cm}^{2} \\
\mathrm{o} / \mathrm{o}\end{array}$ & $\begin{array}{l}(5) \\
1 \cdot 10^{23} \\
3 \cdot 10^{21} \\
99\end{array}$ & $\begin{array}{l}5 \\
1 \cdot 10^{24} \\
1 \cdot 10^{22} \\
7\end{array}$ & $\begin{array}{l}5 \\
3 \cdot 10^{21} \\
1 \cdot 10^{19} \\
70\end{array}$ & $\begin{array}{l}5 \\
1 \cdot 10^{21} \\
3 \cdot 10^{19} \\
15\end{array}$ \\
\hline
\end{tabular}

Tabelle 3. Gemessene, korrigierte und berechnete Ausbeutequotienten sowie zugehörige Lösungen der Parameter.

* nach Vohra ${ }^{18}$, ** nach Aarkrog und Lippert ${ }^{40}$.

*** Rechnung mit den zusätzlichen Ausbeutequotienten $Y_{\mathrm{Cd}-115} / Y_{\mathrm{Zr}-95}$ und $Y_{\mathrm{Eu}-156} / Y_{\mathrm{Zr}-95}$ ändert das Ergebnis nicht.

Der Verlauf der Fehlerkurven (Abb. 2) bei variabler Neutronenenergie $E_{\mathrm{p}}$ zeigt, daß $E_{\mathrm{p}}$ nur schlecht zu bestimmen ist. Deutlich dagegen ist, daß sicherlich nicht $E_{\mathrm{p}}=667 \mathrm{keV}$ zutrifft, weil der betreffende Fehler gegenüber kleineren Neutronenenergien zu groß ist. Es liegt also moderierte Kernspaltung vor. Die häufigste Neutronenenergie kann

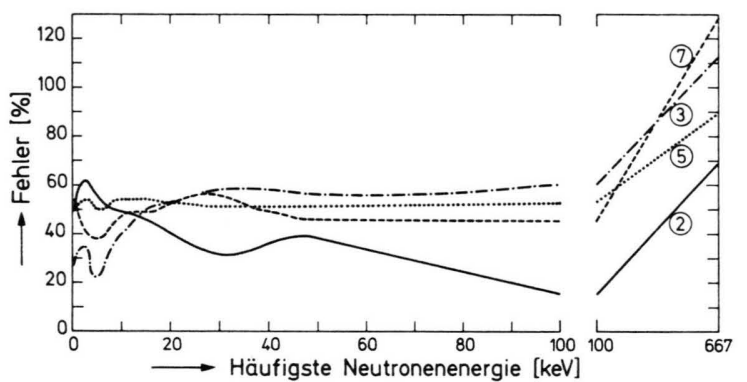

Abb. 2. Der Fehler $F$ nach Gl. (8) für die Tests 2, 3, 5 und 7 als Funktion der häufigsten Neutronenenergie $E_{\mathrm{p}}$.

man mit $5 \mathrm{keV}$ festlegen, obgleich bei Test 2 auch ein höherer Wert von etwa $100 \mathrm{keV}$ möglich ist. Das ist weniger als die von amerikanischen Autoren 14, 15 angenommenen Neutronenenergien, welche bei $20-100 \mathrm{keV}$ liegen; letztere Energien beziehen sich jedoch auf spezielle Kernbombenversuche zur Herstellung von Transuranen, bei denen zur Erzielung höchster Neutronenflüsse die (D,T)-Reaktion eine Rolle spielt; solche Versuche führen sicherlich zu höheren Temperaturen als die chinesischen Kernwaffenversuche.

Die Maxwell-Neutronenflüsse in Tab. 3 liegen zwischen $10^{21}$ bis $10^{24} \mathrm{n} / \mathrm{cm}^{2}$. Es ist nur wenig über Fluenzen bei Kernwaffenversuchen bekannt. BeLL ${ }^{13,15}$ gibt für die Versuche Mike, Par und Barbel Fluenzen von $7 \cdot 10^{24} \mathrm{n} / \mathrm{cm}^{2}$ an, welche man als Maximalwerte auffassen muß. Daran gemessen sind die hier ermittelten Neutronenflüsse teilweise bereits sehr groß. Ein einfacher Zusammenhang zwischen Explosionswert und Neutronenfluß ist nicht evident, was auch von GLAsstone ${ }^{29}$ herausgestellt wird.

Die integralen $14 \mathrm{MeV}$-Neutronenflüsse betragen 0,3-3,0\% der Maxwell-Neutronenflüsse. Das kann wohl kaum als Beitrag durch (D,T)-Reaktion gedeutet werden; vielmehr sieht es so aus, als handele es sich um einen Kompensationseffekt, der das Fehlen energiereicher Neutronen im MaxwellSpektrum ausgleicht. Es wäre aufschlußreich gewesen, den $14 \mathrm{MeV}$-Neutronenfluß der H-Bombe, Test Nr.6, auf die gleiche Weise zu ermitteln. Wegen der geringen spezifischen Aktivität dieser Probe (Tab. 1) konnten jedoch ${ }^{237} \mathrm{U}$ und ${ }^{239} \mathrm{~Np}$ nicht gemessen werden. Der hohe Ausbeutequotient $Y_{\mathrm{Ag}-111} / Y_{\mathrm{Zr}-95}=0,045$ legt die Annahme nahe, daß bei Test 6 die Neutronenenergie höher gewesen sein muß als in den übrigen Tests, wie es für Megatonnenversuche zu erwarten ist ${ }^{45}$.

Der in Tab. 3 gegebene Beitrag des ${ }^{235} \mathrm{U}$ bleibt von kleinen Änderungen der Neutronenflüsse und Änderungen der Neutronenenergie im Bereich $5-100 \mathrm{keV}$ unbeeinflußt. Die Zahlenwerte $Q=N_{\mathrm{U}-235}^{0} /\left(N_{\mathrm{U}-235}^{0}+N_{\mathrm{U}-238}^{0}\right)$ liegen zwischen $7-99 \%$. Aus den großen Werten $Q \sim 85 \%$ kann

45 G. Schumanx, Naturwiss. 54, 6 [1967]. 
man schließen, daß das eingesetzte Spaltmaterial etwa 85-proz. ${ }^{235} \mathrm{U}$ war; zusätzlich muß man annehmen, daß ein eventuell vorhandener NatururanReflektor nicht an der Reaktion beteiligt war oder daß möglicherweise gar kein Natururan-Reflektor vorhanden war. Aus den kleinen Werten $Q \sim 10 \%$ kann man unter Berücksichtigung der Neutronenenergie $E p=5 \mathrm{keV}$ folgern, daß ein Kern aus angereichertem ${ }^{235} \mathrm{U}$ von einem Reflektor aus Natururan umgeben war. Matsunami und Mamuro 46 beschreiben das Auftreten von Natururan in heißen Teilchen nach den chinesischen Tests Nr. 1, 2, 3 und 5 und vermuten die Herkunft aus dem Reflektormaterial. Nach dem in dieser Arbeit verwendeten Arbeitsmodell wird zwischen Kern und Reflektor nicht unterschieden. Das bedeutet: Der in Tab. 3 gegebene Beitrag des ${ }^{235} \mathrm{U}$ ist ein unterer Grenzwert, wenn man ihn nur auf den Bombenkern bezieht. Verallgemeinernd folgt, daß das Spaltmaterial der untersuchten chinesischen Kernwaffentests aus angereichertem ${ }^{235} \mathrm{U}$ bestand, welches mit $5 \mathrm{keV}$-Neutronen gespalten wurde.

Wieviel und welche Ausbeutequotienten braucht man für eine solche Analyse? Tab. 4 zeigt: Die Auswertung von induzierten Radionukliden allein ergibt zu hohe Maxwell-Neutronenflüsse, während aus Spaltprodukten allein der Beitrag des ${ }^{235} \mathrm{U}$ zu hoch ausfällt. Von der Kombination Zr-U-Np-Ag ab ändern zusätzliche Daten das Ergebnis nicht mehr. Für die Fraktionierungskorrektur muß außerdem Ba gemessen werden. Die bemerkenswerte Konstanz der Ergebnisse in Tab. 4 zeigt, daß durch Messung relativ weniger Radionuklide im globalen Fallout die häufigste Neutronenenergie, der integrale Neutronenfluß und das Spaltmaterial des betreffenden Kernwaffenversuchs bestimmt werden können. Bisher war dazu die Messung sehr vieler Transurane erforderlich, die nur aus lokalem Fallout erhalten werden. Mit der hier berichteten Methode wird eine solche Analyse auf den globalen Fallout ausgedehnt.

\section{Anhang}

Definitionen

E Diskrete Neutronenenergie

Ep Häufigste Neutronenenergie einer MaxwellVerteilung

$N(E) \quad$ Häufigkeit von Neutronen der Energie $E$ im Maxwellschen Neutronenspektrum

$s(E) \quad$ Wirkungsquerschnitt

$S \quad$ Mittlerer Wirkungsquerschnitt für ein Maxwellsches Neutronenspektrum

$S_{\mathrm{i}}^{\mathrm{abs}}, S_{\mathrm{i}}^{\mathrm{n}, \mathrm{f}}, S_{\mathrm{i}}^{\mathrm{n}, \gamma}, S_{\mathrm{i}}^{\mathrm{n}, 2 \mathrm{n}}$

Mittlere Wirkungsquerschnitte des Spaltmaterials i für Absorption, Kernspaltung, $(\mathrm{n}, \gamma)$ - und (n, 2n)-Reaktion

$\sigma_{\mathrm{i}}^{\mathrm{abs}}, \sigma_{\mathrm{i}}^{\mathrm{n}, \mathrm{f}}, \sigma_{\mathrm{i}}^{\mathrm{n}, \gamma}, \sigma_{\mathrm{i}}^{\mathrm{n}, 2 \mathrm{n}}$

Analoge Wirkungsquerschnitte für $14 \mathrm{MeV}$.

Neutronen

$\Phi t, \varphi \tau$ Fluenz, zeitintegrierter Neutronenfluß der Maxwellneutronen, bzw. 14 MeV-Neutronen

$Y_{\mathrm{U}-235}^{\mathrm{k}}, Y_{\mathrm{U}-238}^{\mathrm{k}}, y_{\mathrm{U}-235}^{\mathrm{k}}, y_{\mathrm{U}-238}^{\mathrm{k}}$

Spaltausbeute des Spaltprodukts $\mathrm{k}$ für ${ }^{235} \mathrm{U}$ - bzw. ${ }^{238} \mathrm{U}$-Spaltung mit MaxwellNeutronen bzw. $14 \mathrm{MeV}$-Neutronen

$N_{\mathrm{i}}^{t}, N_{\mathrm{i}}^{0} \quad$ Zahl der Atome des Nuklids i zur Zeit $t$ bzw. $t=0$

\section{Mittlere Wirkungsquerschnitte}

Die Energieverteilung der Neutronen wird bei der Rechnung berücksichtigt, indem anstelle energieabhängiger Wirkungsquerschnitte $s(E)$ die mittleren Wirkungsquerschnitte $S$ benutzt werden, wie Gl. (4) definiert:

$$
S=\int_{0}^{\infty} s(E) n(E) \mathrm{d} E / \int_{0}^{\infty} N(E) \mathrm{d} E .
$$

\begin{tabular}{lccc}
\hline \multicolumn{1}{c}{ Kombination } & $\begin{array}{c}\Phi t \\
\mathrm{n} / \mathrm{cm}^{2}\end{array}$ & $\begin{array}{c}\varphi \tau \\
\mathrm{n} / \mathrm{cm}^{2}\end{array}$ & $\begin{array}{c}N_{235}^{0} /\left(N_{235}^{0}+N_{238}^{0}\right) \\
\%\end{array}$ \\
\hline $\mathrm{Zr}-\mathrm{Ag}-\mathrm{Cd}-\mathrm{Eu}$ & $1 \cdot 10^{21}$ & $1 \cdot 10^{20}$ & 60 \\
$\mathrm{Zr}-\mathrm{U}-\mathrm{Np}$ & $1 \cdot 10^{23}$ & $3 \cdot 10^{20}$ & 15 \\
$\mathrm{Zr}-\mathrm{U}-\mathrm{Np}-\mathrm{Ag}$ & $1 \cdot 10^{21}$ & $3 \cdot 10^{19}$ & 15 \\
$\mathrm{Zr}-\mathrm{U}-\mathrm{Np}-\mathrm{Ag}-\mathrm{Cd}$ & $1 \cdot 10^{21}$ & $3 \cdot 10^{19}$ & 15 \\
$\mathrm{Zr}-\mathrm{U}-\mathrm{Np}-\mathrm{Ag}-\mathrm{Cd}-\mathrm{Eu}$ & $1 \cdot 10^{21}$ & $3 \cdot 10^{19}$ & 15 \\
\hline
\end{tabular}

Tab. 4. Parameter in Abhängigkeit von verschiedenen Ausbeutequotienten Test 7 .

46 T. Matsunami u. T. Mamuro, Nature 218, 555 [1968]. 
Für die numerische Auswertung von Gl. (4) benötigt man außer den energieabhängigen Wirkungsquerschnitten 47 und Spaltausbeuten ${ }^{44}$ auch das Neutronenspektrum. Für die Spaltneutronendichte $\left(\mathrm{n} / \mathrm{cm}^{3}\right)$ gilt eine Maxwell-Verteilung 48,49 :

$$
N(E)=\text { const. } E^{1 / 2} \cdot e^{-E / 2 E_{\mathrm{p}}} .
$$

Diese Anwendung von Gl. (5) ist eine Näherung für die wirkliche Energieverteilung. Gl. (5) hat ein Häufigkeitsmaximum der Neutronenenergie und ein langsames Abklingen der Häufigkeit von hochenergetischen Neutronen. Dasselbe leistet auch eine Maxwell-Verteilung der Spaltneutronenflußdichte $\left(\mathrm{n} / \mathrm{cm}^{2}\right)$. Da hier die häufigste Neutronenenergie des Spektrums bestimmt werden soll - eine vollständige Energieverteilung kann nur direkt gemessen werden - ist es von geringer Bedeutung, ob die Maxwell-Verteilung der Neutronendichte oder der Neutronenflußdichte benutzt wird; bei der Rechnung resultiert derselbe Zahlenwert für $E p$.

\section{Verbrauch des Spaltmaterials}

Weil das Arbeitsmodell zwei aufeinander folgende Kernspaltungsreaktionen vorsieht, ist die jeweils vorhandene Menge des Spaltmaterials von zwei $e$-Funktionen abhängig:

$$
\begin{aligned}
N_{\mathrm{U}-235}^{t}=N_{\mathrm{U}-235}^{0} \cdot \exp \left\{-S_{\mathrm{U}-235}^{\mathrm{abs}} \cdot \Phi t\right\} \\
\cdot \exp \left\{-\sigma_{\mathrm{U}-235}^{\mathrm{abs}} \cdot \varphi \tau\right\} .
\end{aligned}
$$

Für ${ }^{238} \mathrm{U}$ gilt Gl. (6) analog.

\section{Bildung von Radioisotopen}

Die nach Bateman 50 abgeleiteten Gleichungen über die Bildung von Spaltprodukten und induzierten Radionukliden aus angereichertem ${ }^{235} \mathrm{U}$ werden hier abgekürzt formuliert:

$$
\begin{aligned}
N_{\mathrm{i}}^{t} & =N_{\mathrm{U}-238}^{0}\left(\pi_{\mathrm{S}}^{238}+\pi_{\sigma}^{238} \cdot \exp \left\{-S_{\mathrm{U}-238}^{\mathrm{abs}} \cdot \Phi t\right\}\right) \\
& +N_{\mathrm{U}-235}^{0}\left(\pi_{\mathrm{S}}^{235}+\pi_{\sigma}^{235} \cdot \exp \left\{-S_{\mathrm{U}-235}^{\mathrm{abs}} \cdot \Phi t\right\}\right) .
\end{aligned}
$$

$\pi$ stellt darin Terme dar, die verschieden sind, jedoch weitgehende Analogie besitzen. Die vier $\pi$-Terme pro Radionuklid erhält man aus der jeweils angegebenen Definition durch Indexaustausch.

47 K. Parker, United Kingdom Authority, Atomic Weapons Research Establishment, Aldermaston, Bks., AWRE $-0-79 / 63$; AWRE-0-82/63; AWRE-0-30/64.

48 J. Terrell, Phys. Rev. 113, 527 [1959].

49 U. S. Atomic Energy Comm., New York, Argonne National Lab., ANL-5800, 2nd Edition [1963].

50 G. Friedla ender, J. W. Kennedy u. J. M. Miller, Nuclear and Radiochemistry, 2nd Edition, John Wiley \& Sons, Inc., New York 1964, S. 80.
$\pi$-Definition für Spaltprodukte, Beispiel ${ }^{95} \mathrm{Zr}$ :

$\pi_{\mathrm{S}}^{238}=\frac{S_{\mathrm{U}-238}^{\mathrm{n} . \mathrm{f}} Y_{\mathrm{U}-238}^{\mathrm{Zr}-95}}{S_{\mathrm{U}-238}^{\mathrm{abs}}} \cdot\left(1-\exp \left\{-S_{\mathrm{U}-238}^{\mathrm{abs}} \cdot \Phi t\right\}\right)$

$\pi_{\mathrm{S}}^{235}$, Austausch : $238 \rightarrow 235$;

$\pi_{\sigma}^{238}$, Austausch: $\Phi t \rightarrow \varphi \tau, S \rightarrow \sigma ;$

$\pi_{\sigma}^{235}$, Austausch : $238 \rightarrow 235, S \rightarrow \sigma, \Phi t \rightarrow \varphi \tau$.

Zusätzlich ist der Index Zr-95 gegen den Index des betreffenden Spaltprodukts auszutauschen.

$\pi$-Definition für ${ }^{239} \mathrm{~Np}$ :

$$
\begin{aligned}
\pi_{\mathrm{S}}^{238}=S_{\mathrm{U}-238}^{\mathrm{n}, \gamma} \cdot\left(\frac{\exp \left\{-S_{\mathrm{U}-238}^{\mathrm{abs}} \cdot \Phi t\right\}}{S_{\mathrm{U}-238}^{\mathrm{abs}}-S_{\mathrm{U}-239}^{\mathrm{abs}}}\right. & \\
& \left.+\frac{\exp \left\{-S_{\mathrm{U}-239}^{\mathrm{abs}} \cdot \Phi t\right\}}{S_{\mathrm{U}-239}^{\mathrm{abs}}-S_{\mathrm{U}-238}^{\mathrm{abs}}}\right)
\end{aligned}
$$

$\pi_{\mathrm{S}}^{235}=0 ; \quad \pi_{\sigma}^{235}=0 ;$

$\pi_{\sigma}^{238}$, Austausch: $\Phi t \rightarrow \varphi \tau, S \rightarrow \sigma$.

$\pi$-Definition für ${ }^{237} \mathrm{U}$ :

$$
\begin{aligned}
\pi_{\mathrm{S}}^{238}=S_{\mathrm{U}-238}^{\mathrm{n}, 2 \mathrm{n}} \cdot\left(\frac{\exp \left\{-S_{\mathrm{U}-238}^{\mathrm{abs}} \cdot \Phi t\right\}}{S_{\mathrm{U}-238}^{\mathrm{abs}}-S_{\mathrm{U}-237}^{\mathrm{abs}}}\right. & \\
& \left.+\frac{\exp \left\{-S_{\mathrm{U}-237}^{\mathrm{abs}} \cdot \Phi t\right\}}{S_{\mathrm{U}-237}^{\mathrm{abs}}-S_{\mathrm{U}-238}^{\mathrm{abs}}}\right) ;
\end{aligned}
$$

$\pi_{238}^{\sigma}$, Austausch: $\Phi t \rightarrow \varphi \tau, S \rightarrow \sigma ;$

$$
\begin{aligned}
\pi_{\mathrm{S}}^{235}=S_{\mathrm{U}-235}^{\mathrm{n}, \gamma} S_{\mathrm{U}-236}^{\mathrm{n}, \gamma} & \cdot\left(\frac{\exp \left\{-S_{\mathrm{U}-235}^{\mathrm{abs}} \cdot \Phi t\right\}}{\left(S_{\mathrm{U}-237}^{\mathrm{abs}}-S_{\mathrm{U}-235}^{\mathrm{abs}}\right)\left(S_{\mathrm{U}-236}^{\mathrm{abs}}-S_{\mathrm{U}-235}^{\mathrm{abs}}\right)}\right. \\
& +\frac{\exp \left\{-S_{\mathrm{U}-236}^{\mathrm{abs}} \cdot \Phi t\right\}}{\left(S_{\mathrm{U}-237}^{\mathrm{abs}}-S_{\mathrm{U} \cdot 236}^{\mathrm{abs}}\right)\left(S_{\mathrm{U}-235}^{\mathrm{abs}}-S_{\mathrm{U}-236}^{\mathrm{abs}}\right)} \\
& \left.+\frac{\exp \left\{-S_{\mathrm{U}-237}^{\mathrm{abs}} \cdot \Phi t\right\}}{\left(S_{\mathrm{U}-236}^{\mathrm{abs}}-S_{\mathrm{U}-237}^{\mathrm{abs}}\right)\left(S_{\mathrm{U}-235}^{\mathrm{abs}}-S_{\mathrm{U}-237}^{\mathrm{abs}}\right)}\right)
\end{aligned}
$$

$\pi_{235}^{\sigma}$, Austausch: $\Phi t \rightarrow \varphi \tau, S \rightarrow \sigma$.

\section{Kriterium für Übereinstimmung}

$$
F=\sqrt{\sum_{1}^{n}\left(\text { l-Rechnungswert/Meßwert) }{ }^{2} .\right.}
$$

Herrn Prof. Dr. F. Strassmann gehört mein Dank für die vielfältige Arbeitsmöglichkeit im Institut. Herrn Prof. Dr. G. Herrmans danke ich für Anregungen im Verlaufe der Arbeit. Die Fraunhofer-Gesellschaft zur Förderung der angewandten Forschung e.V. München stellte die Mittel für die Arbeit zur Verfügung, die Lufthansa Frankfurt/Main ermöglichte die Probenahme des Fallout. Beiden Institutionen gebührt mein Dank ebenso wie Frau E. KreBs und Frl. K. WAChNer für die Ausführung der experimentellen Arbeit. Die Programmierung und Rechnung auf der Siemens 2002 im Rechenzentrum der Universität Mainz ist das Verdienst von Herrn Dipl. Chem. D. Jung, für dessen freundliche Mitarbeit ich herzlich danke. 